\title{
CDISC SEND Pregnancy Findings Result Terminology
}

National Cancer Institute

\section{Source}

National Cancer Institute. CDISC SEND Pregnancy Findings Result Terminology. NCI

Thesaurus. Code C124323.

Terminology associated with the pregnancy findings result codelist of the Clinical Data Interchange Standards Consortium (CDISC) Standard for the Exchange of Non-clinical data (SEND). 